\title{
Free energy versus internal energy potential for heavy quark systems at finite temperature
}

\author{
Taesoo Song, ${ }^{\mathrm{a}, \mathrm{e}}$, Su Houng Lee ${ }^{\mathrm{b}}$, Kenji Morita ${ }^{\mathrm{a}, \mathrm{c}, \mathrm{d}}$, Che Ming Ko ${ }^{\mathrm{f}}$ \\ ${ }^{a}$ Frankfurt Institute for Advanced Studies, Ruth-Moufang-Str. 1, D-60438 Frankfurt am Main, Germany \\ ${ }^{b}$ Department of Physics and Institute of Physics and Applied Physics, Yonsei University, Seoul 120-749, Korea \\ ${ }^{c}$ Institute of Theoretical Physics, University of Wroclaw, PL-50204 Wroctaw, Poland \\ ${ }^{d}$ Yukawa Institute for Theoretical Physics, Kyoto University, Kyoto 606-8502, Japan \\ ${ }^{e}$ Institute for Theoretical Physics, Johann Wolfgang Goethe Universität, Max-von-Laue-Str. 1, D-60438, Frankfurt am Main, Germany \\ ${ }^{f}$ Cyclotron Institute and Department of Physics and Astronomy, Texas AEM University, College Station, Texas 77843, U.S.A.
}

\begin{abstract}
The strength of the $J / \psi$ wave function at origin $|\psi(0)|$ is calculated by using the finite temperature QCD sum rule with the gluon condensates determined from the lattice QCD. The result is compared with that from a potential model based on the static free or internal energy obtained from lattice calculations. We find that the $|\psi(0)|$ decreases with temperature around the deconfinement transition temperature both in the QCD sum rule and in the potential model with the free energy potential. Our result thus suggests that the free energy potential from lattice calculations is appropriate for describing the properties of charmonia at finite temperature.
\end{abstract}

Keywords: $J / \psi$ suppression, QCD sum rule, Heavy quark potential

\section{Introduction}

Since Matsui and Satz suggested the $J / \psi$ suppression as a signature for the formation of a quark-gluon plasma (QGP) in relativistic heavy-ion collisions [1], numerous theoretical and experimental studies have been carried out on this subject. Experimental data from the Super Proton Synchrotron (SPS) to the Large Hadron Collider (LHC) have indeed shown a suppression of $J / \psi$ production. However, it is not clear whether $J / \psi$ dissolves immediately above the critical temperature $T_{c}[2,3,4,5]$ or remains bounded at higher temperatures $[6,7,8]$, because both scenarios can explain the experimental data.

Lattice QCD calculations have provided with great precision the free energy $F$ of a pair of static heavy quark $Q$ and its antiquark $\bar{Q}$ in a hot QCD matter and also their internal energy $U$ by adding the entropy contribution to the free energy [9]. Once the heavy quark potential is given, the properties of $J / \psi$ at finite temperature can be obtained by solving the Schrödinger equation. If the free energy is used as the potential in the Schrödinger equation for the $Q \bar{Q}$ system, the $J / \psi$ dissolves slightly above $T_{c}$, while it remains bounded up to almost $2 T_{c}$ if the internal energy is used as the potential. Due to the sharp rise of the internal energy potential with increasing interquark distance, the resulting $J / \psi$ wave function is more localized at the origin than that from the free energy potential.

Since the $J / \psi$ wave function at the origin $|\psi(0)|$ is the non-relativistic limit of the overlap of a $J / \psi$ with the charm vector current $\bar{c} \gamma_{\mu} c$, one may compare the result from the potential model calculations with that determined from the current correlation function. In this proceedings, we highlight our results on the temperature dependence of the $|\psi(0)|$ obtained from the QCD sum rule and that obtained by solving the Schrödinger equation with the free energy and internal energy potentials. More details can be found in Ref. [10]. 


\section{The QCD sum rule approach}

We start with the correlation function of the charm vector current $J_{\mu}=\bar{c} \gamma_{\mu} c$,

$$
\Pi_{\mu v}(q)=i \int d^{4} x e^{i q x}\left\langle T\left[J_{\mu}(x) J_{v}(0)\right]\right\rangle .
$$

Defining the charm quark pair polarization function $\Pi(q)=-\Pi_{\mu}^{\mu}(q) /\left(3 q^{2}\right)$, we find that the $J / \psi$ contribution to its imaginary part is given by

$$
\operatorname{Im} \Pi(s)=f_{0} \delta\left(s-m_{J / \psi}^{2}\right),
$$

where the residue of the charmonium correlator

$$
f_{0}=\frac{12 \pi}{m_{J / \psi}}|\psi(0)|^{2}
$$

is related to the dielectron decay width of $J / \psi$ via $\Gamma_{J / \psi}^{e^{+} e^{-}}=\frac{16 \pi \alpha^{2} e_{Q}^{2}}{m_{J / \psi}^{2}}|\psi(0)|^{2}$. Using the empirical value $\Gamma_{J / \psi}^{e^{+} e^{-}}=5.55$ $\mathrm{KeV}$ for a $J / \psi$ in the vacuum [11], we obtain the value $|\psi(0)|=0.211 \pm 0.04 \pm 0.05 \mathrm{GeV}^{3 / 2}$ for the overlap of the charm vector current with a free $J / \psi$ or the wave function of a free $J / \psi$ at the origin, where the uncertainties are due to relativistic corrections and higher-order QCD effects.

On the other hand, the residue of the charmonium correlator $f_{0}$ can be reliably calculated using the QCD sum rule method $[12,13]$. Here we use the Borel transformed sum rule, which suppresses contributions from the continuum, to calculate the $f_{0}$ as it gives more reliable results with respect to changes in the continuum [14]. The generalization of the Borel sum rule to finite temperature is well founded as the operator product expansion (OPE) is well known up to dimension 4 operators by using the energy density and pressure of hot QCD matter from lattice calculations. The Borel sum rule for the $f_{0}$ only involves the strength at the pole, which is an integrated quantity, and thus does not depend on details of the spectral density. Hence, we concentrate on the temperature dependence of $f_{0}$.

Specifically, the OPE for the charm quark pair polarization function $\Pi(q)$ is equated to the spectral density via the Borel transformed dispersion relation

$$
\mathcal{M}\left(M^{2}\right)=\int_{0}^{\infty} d s e^{-s / M^{2}} \operatorname{Im} \Pi(s)
$$

after neglecting the thermal factor $\tanh [s / 2 T]$ in the spectral density as its correction to the charmonium and the continuum is negligible up to the temperature of $1.1 T_{c}[15,16]$. For the spectral density, we use the conventional "pole+continuum" ansatz $\operatorname{Im} \Pi(s)=\operatorname{Im} \Pi^{J / \psi}(s)+\theta\left(s-s_{0}\right) \operatorname{Im} \Pi^{\text {pert }}(s)$, where the first term is given by the pole form in Eq. (2) and the second term is the perturbative correlation function with $s_{0}$ being the effective continuum threshold.

The Borel transformed OPE and its temperature dependence up to dimension 4 operators are

$$
\mathcal{M}\left(M^{2}\right)=e^{-v} \pi A(v)\left[1+\alpha_{s}\left(M^{2}\right) a(v)+b(v) \phi_{b}(T)+c(v) \phi_{c}(T)\right]
$$

with $v=4 m_{c}^{2} / M^{2}$ being a dimensionless scale parameter. The Wilson coefficients $A(v), a(v), b(v)$, and $c(v)$ are summarized in Ref. [17], where the temperature dependence of the scalar and twist-2 gluon condensates $\phi_{b}$ and $\phi_{c}$ are also given.

Using the OPE side of Eq. (5), we can express the residue $f_{0}$ as

$$
f_{0}=e^{m_{J / \psi}^{2}\left(M^{2}\right) / M^{2}}\left[\mathcal{M}^{\mathrm{OPE}}\left(M^{2}\right)-\mathcal{M}^{\mathrm{cont}}\left(M^{2} ; s_{0}\right)\right],
$$

where the $J / \psi$ mass is given by

$$
m_{J / \psi}^{2}=-\frac{\frac{\partial}{\partial\left(1 / M^{2}\right)}\left(\mathcal{M}^{\mathrm{OPE}}\left(M^{2}\right)-\mathcal{M}^{\mathrm{cont}}\left(M^{2} ; s_{0}\right)\right)}{\mathcal{M}^{\mathrm{OPE}}\left(M^{2}\right)-\mathcal{M}^{\mathrm{cont}}\left(M^{2} ; s_{0}\right)} .
$$

We determine $s_{0}$ by requiring $|\psi(0)|=\sqrt{m_{J / \psi} f_{0} /(12 \pi)}$ to be least sensitive to $M^{2}$. The technical detail can be found in Ref. [18] for the determination of $m_{J / \psi}$ at finite temperature. We have confirmed that the $M^{2}$ dependence of $|\psi(0)|$ is quite similar to that of $m_{J / \psi}$. We note that the required value for $s_{0}$ differs slightly from the case in which the Borel curve for $m_{J / \psi}$ is optimized. 


\section{The Schrödinger equation approach and results}

To find out which potential between a charm and anticharm quark pair correctly reproduces the $|\psi(0)|$ obtained from the QCD sum rule, we numerically solve the Schrödinger equation for a charm and anticharm pair:

$$
\left[2 m_{c}-\frac{1}{m_{c}} \nabla^{2}+V(r, T)\right] \psi(r, T)=M \psi(r, T),
$$

where $m_{c}=1.25 \mathrm{GeV}$ is the bare mass of charm quark and $\psi(r, T)$ is the charmonium wave function at temperature $T$. Introducing the potential $V(r=\infty, T)$ at infinitely large distance, the Schrödinger equation is modified to [19, 20]

$$
\left[-\frac{\nabla^{2}}{m_{c}}+\widetilde{V}(r, T)\right] \psi(r, T)=-\varepsilon \psi(r, T)
$$

where $\widetilde{V}(r, T) \equiv V(r, T)-V(r=\infty, T)$ vanishes at infinitely large distance, and $\varepsilon=2 m_{c}+V(r=\infty, T)-M$ is the binding energy of a $J / \psi$ at temperature $T$. For the heavy quark potential, we use either the free energy between a heavy quark-antiquark pair that is extracted from lattice calculations [21, 20, 9] or the more attractive one based on the internal energy by adding the contribution from the entropy density [9].
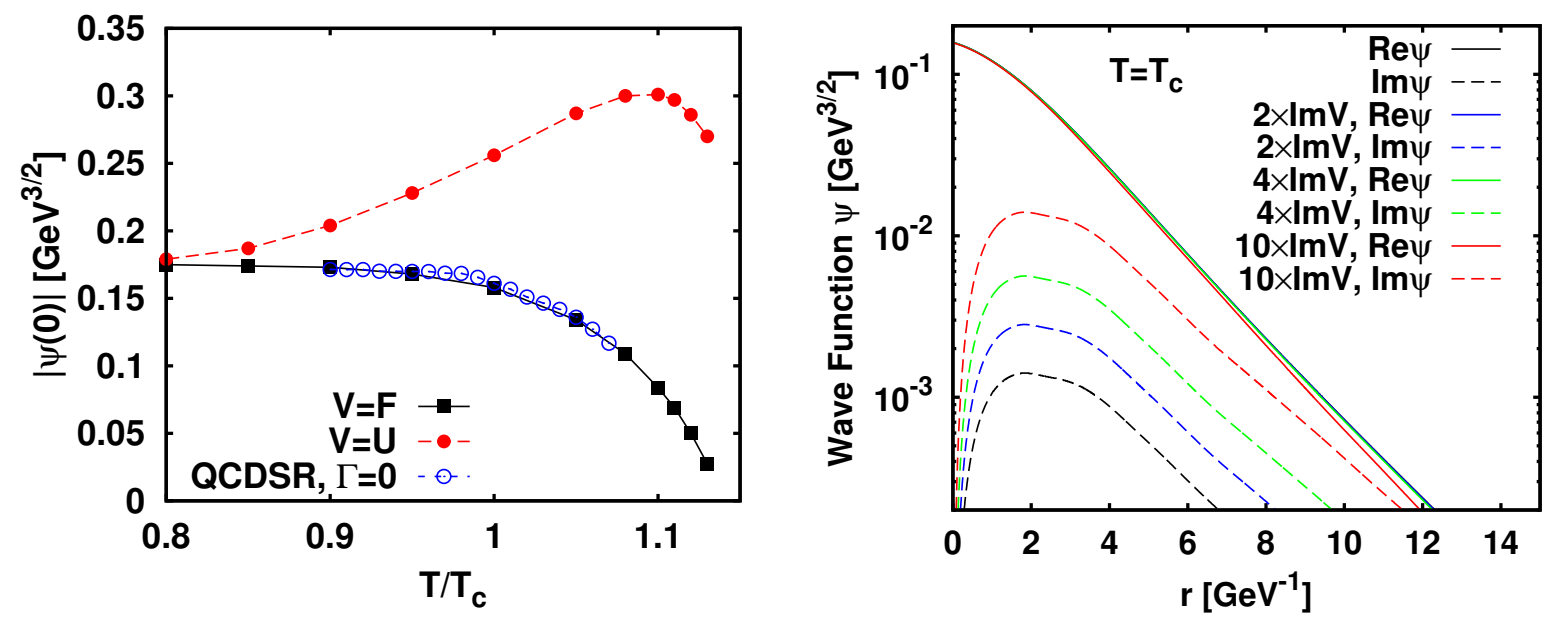

Figure 1. Left : Temperature dependence of $|\psi(0)|$ obtained from the potential model based on the free energy (filled squares) or the internal energy (filled circles) together with that from the QCD sum rule (open circles). Right: Wave function of $J / \psi$ for different values of the imaginary part of the potential.

In the left panel of Fig. 1, we show the temperature dependence of $|\psi(0)|$ obtained by solving the Schrödinger with the internal or the free energy potential and also from the QCD sum rule in the case of a vanishing width. With the internal energy as the potential, the strength of $|\psi(0)|$ increases by almost a factor of two at slightly above the critical temperature, reminiscent of the "overshooting" effect discussed in Ref. [3] that adding the entropy contribution would lead to a more attractive heavy quark potential near $T_{c}$ than at zero temperature. On the other hand, the strength of $|\psi(0)|$ decreases monotonically with temperature when the free energy is used as the potential, strikingly similar to the result from QCD sum rules in both its behavior and values. Our results thus indicate that using the free energy potential from lattice calculations in the Schrödinger equation for a charm and anticharm quark pair correctly describes the non-relativistic wave function of a $J / \psi$ at finite temperature. Such a potential also reproduces the mass of $J / \psi$ at finite temperature from the QCD sum rule analysis [10].

Above the critical temperature, one expects the emergence of an imaginary part in the $Q \bar{Q}$ potential [22]. As an illustration, we examine the effect of the imaginary part by including the imaginary potential from the Hard Thermal 
Loop (HTL) calculations [22, 23]. Results on the real and imaginary parts of the wave function are shown in the right panel of Fig. 1 for temperature at $T=T_{c}$. It is seen that for both the free and internal energy potentials, this only changes the value of $|\psi(0)|^{2}$ by less than $2 \%$ even after increasing the magnitude of the imaginary part by a factor of ten. Since the effect of imaginary potential remains small at other temperatures considered in the present study, it thus does not affect our results.

\section{Conclusion}

Because using the free energy calculated in the lattice QCD as the heavy quark potential leads to a $J / \psi$ dissociation temperature that is only slightly above $T_{c}$ [24], all charmonium states would essentially melt in the quark-gluon plasma. In this case, the observed $J / \psi$ 's in relativistic heavy ion collisions are then either from initial hard collisions that occur outside the QGP or from the recombination of charm and anticharm quarks in the QGP during hadronization.

\section{Acknowledgements}

This work was supported in part by the Korean Research Foundation under Grant Nos. KRF-2011-0020333 and KRF-2011-0030621, the Yukawa International Program for Quark-Hadron Sciences at Kyoto University and the Grant-in-Aid for Scientific Research from JSPS No. 24540271, the Polish Science Foundation (NCN), under Maestro grant 2013/10/A/ST2/00106, and the DFG BR 4000/3-1 as well as the US National Science Foundation under Grant No. PHY-1068572, the US Department of Energy under Contract No. DE-FG02-10ER41682 within the framework of the JET Collaboration, and the Welch Foundation under Grant No. A-1358.

\section{References}

[1] T. Matsui and H. Satz, Phys. Lett. B 178, 416 (1986).

[2] A. Mocsy and P. Petreczky, Phys. Rev. Lett. 99, 211602 (2007).

[3] A. Dumitru, Y. Guo, A. Mocsy and M. Strickland, Phys. Rev. D 79, 054019 (2009).

[4] P. Gubler, K. Morita and M. Oka, Phys. Rev. Lett. 107, 092003 (2011).

[5] H. T. Ding et al., Phys. Rev. D 86, 014509 (2012).

[6] M. Asakawa and T. Hatsuda, Phys. Rev. Lett. 92, 012001 (2004).

[7] S. Datta et al., Phys. Rev. D 69, 094507 (2004).

[8] H. Ohno et al. [WHOT-QCD Collaboration], Phys. Rev. D 84, 094504 (2011).

[9] O. Kaczmarek, Eur. Phys. J. C 61, 811 (2009).

[10] S. H. Lee, K. Morita, T. Song, C. M. Ko, Phys. Rev. D 89, 094015 (2014).

[11] J. Beringer et al. (PDG), Phys. Rev. D86, 010001 (2012).

[12] M. A. Shifman, A. I. Vainshtein and V. I. Zakharov, Nucl. Phys. B 147, 385 (1979); B 147, 448 (1979).

[13] L.J. Reinders, H. Rubinstein and S. Yazaki, Phys. Rept. 127, 1 (1985).

[14] R. A. Bertlmann, Nucl. Phys. B 204, 387 (1982).

[15] K. Morita and S. H. Lee, Phys. Rev. Lett. 100, 022301 (2008).

[16] K. Morita and S. H. Lee, Phys. Rev. C 77, 064904 (2008).

[17] K. Morita and S. H. Lee, Phys. Rev. D 82, 054008 (2010).

[18] K. Morita and S. H. Lee, Phys. Rev. C85, 044917 (2012).

[19] F. Karsch et al., Z. Phys. C 37, 617 (1988).

[20] H. Satz, J. Phys. G 32, R25 (2006).

[21] S. Digal et al., Eur. Phys. J. C 43, 71 (2005).

[22] M. Laine, O. Philipsen, P. Romatschke and M. Tassler, JHEP 0703, 054 (2007).

[23] P. Petreczky, C. Miao and A. Mocsy, Nucl. Phys. A 855, 125 (2011).

[24] O. Kaczmarek and F. Zantow, PoS LAT 2005, 192 (2006). 